\title{
Association Between Age and Short-Term Patient Reported Pain Scores After Complex Spinal Fusion for Adult Deformity Correction: Is Perception of Pain Different?
}

Aladine A. Elsamadicy M.D. ${ }^{1}$, Megan Lee, B.S. ${ }^{1}$, Andrew Koo B.S. ${ }^{1}$, Adam J. Kundishora M.D. ${ }^{1}$, Joaquin Camara-Quintana M.D. ${ }^{1}$, Tariq Qureshi мввs, мвА ${ }^{1}$, Luis Kolb м.D. ${ }^{1}$, Maxwell Laurans, M.D., M.B.A. ${ }^{1}$, Khalid Abbed M.D. ${ }^{1}$, Isaac O. Karikari M.D. ${ }^{2}$

${ }^{1}$ Department of Neurosurgery, Yale University School of Medicine, New Haven, CT

${ }^{2}$ Department of Neurosurgery, Duke University Medical Center, Durham, NC

${ }^{*}$ Corresponding Author: Aladine A. Elsamadicy, M.D., Department of Neurosurgery, Yale University School of Medicine, 333 Cedar Street, New Haven, CT 06520; Email: aladine.elsamadicy@yale.edu

Received: April 12, 2019; Accepted: April 18, 2019; Published: May 11, 2019;

\begin{abstract}
OBJECTIVE: Complex spinal deformities requiring $\geq 5$ fusion levels is challenging, and significantly impacts the quality of life for the patients. Patient reported pain scores are becoming increasingly important as it sheds insight into the patient's perception of health stat, as well as serving as a proxy of satisfaction for patients with spine deformity undergoing corrective surgery. However, with an aging population, the impact of age on perception of pain before and after undergoing complex deformity correction is understudied. The aim of this study was to evaluate whether there was an association between age and patient-reported pain scores after complex spinal fusions.

METHODS: The medical records of 92 adult ( $\geq 18$ years old) spine deformity patients undergoing elective, primary complex spinal fusion ( $\geq 5$ levels) for deformity correction at a major academic institution from 2010 to 2015 were reviewed. Patients were grouped by age: young ( $<65$ years old) and older ( $\geq 65$ years old). We identified $43(46.7 \%)$ patients $\geq 65$ years old and $49(53.3 \%)<65$ years old (Elderly: $n=43$ vs. Young: $n=49)$. Patient demographics, comorbidities, intraoperative and postoperative complication rates were collected for each patient. Inpatient patient-reported pain scores and ambulatory status were also collected. The primary outcome of this study was patient-reported pain scores.

RESULTS: Patient demographics and comorbidities were slightly different between both cohorts, with the Elderly cohort having a greater proportion of patients with hypertension, hyperlipidemia, and osteoarthritis, Table 1. The median number of fusion levels operated, length of surgery, estimated blood loss, and complication rates were similar between both cohorts, Table 2. Moreover, the post-operative complication profiles between the cohorts were also similar, except for the Elderly having a higher rate of post-operative delirium $(16.3 \%$ vs. $0.0 \%)$, Table 3. Baseline $(p=0.1885)$, First- $(p=0.3331)$ and Last- $(\mathrm{p}=0.1990)$ post-operative patient reported pain scores were similar between cohorts, Table 4 . However, the Elderly cohort trended to have a greater reduction of pain from baseline to Last pain score, compared to the Young cohort (Elderly: $-2.4 \pm 4.1$ vs. Young: $-0.69 \pm 4.5$, $p=0.0556$ ), Table 4. While ambulation immediately before discharge was similar in both groups, the Elderly cohort had significantly fewer ambulatory steps on the first post-operative ambulatory day compared to Young cohort (Elderly: $40.2 \pm 63.4$ vs. Young: $106.0 \pm 134.6, p=0.0042$ ), Table 4 .
\end{abstract}

CONCLUSIONS: Our study suggests that age may have an impact in patient perception of pain and improvement after complex spinal fusions ( $\geq 5$ levels). Consideration of patients' age may facilitate tailored pain management and physical therapy regimens in deformity patients undergoing correction surgery.

\section{INTRODUCTION}

In the last decade, patient-reported outcomes (PRO) measures have grown to be a significant proxy for overall quality of care [1]. In spine surgery, many hospitals use a variety of PRO metrics as a mean to qualify surgical effectiveness and satisfaction [2,3]. One particular PRO that has been shown to have the greatest influence on patients' functional status and satisfaction has been pain scores. Pain is the driving factor for most patients to undergo elective deformity correction surgery. In a study by author et al. of xx patients undergoing deformity surgery, demonstrated that $80 \%$ of patients presenting to clinic have pain as a driving factor. Complex spine surgery involving 5-levels and greater impact patients significantly, however it has provided tremendous quality of life, functionality and pain reduction. Identifying risk factors that influence perception of pain after deformity correction surgery is necessary to better overall quality of care.

With an aging population, there has been an expansion of patients presenting with adult spine deformity (ASD) [4,5]. Elderly patients undergoing complex spine deformity surgery have unique surgical challenges due to increasing medical comorbidities, fragility and physiological changes associated with age. Similarly, geriatric patients present with a varying perception of pain, functionality and disability. 
Previous studies have demonstrated mixed associations with age and pain scores, with some demonstrating a negative correlated with age $[6,7]$, greater pain with increasing age [8-10], and even no correlation at all [11-13]. However, previous studies have mostly looked at spinal fusions involving less than 3 levels, with a paucity of data in complex spinal fusion ( $\geq 5$ levels) patients.

The aim of this study was to evaluate whether there was an association between age and patient-reported pain scores after complex spinal fusions.

\section{METHODS}

The medical records of 92 adult ( $\geq 18$ years old) spine deformity patients undergoing elective, primary complex spinal fusion $(\geq 5$ levels) for deformity correction at a major academic institution from 2010 to 2015 were reviewed. Institutional review board approval was obtained prior to study initiation. Inclusion criteria included patients with 1) available demographics and treatment; 2) who underwent an elective, primary complex spinal fusion ( $\geq 5$ levels) for deformity correction; and 3) who had baseline and post-operative patient reported pain scores. Patients were grouped by age: young $(<65$ years old) and older ( $\geq 65$ years old). We identified 43 patients $(46.7 \%) \geq 65$ years old and 49 patients $(53.3 \%)<65$ years old (Elderly: $n=43$ vs. Young: $n=49$ ). Patient demographics, comorbidities, intraoperative and postoperative complication rates were collected for each patient. Inpatient patient-reported pain scores and ambulatory status were also collected. The primary outcome of this study was the difference in patient-reported pain scores between elderly and young patients undergoing complex spine deformity surgery.

Baseline characteristics and demographic variables evaluated included patient age, sex, and body mass index (BMI). Comorbidities included depression, anxiety, chronic obstructive pulmonary disease (COPD), diabetes, congestive heart failure (CHF), coronary artery disease (CAD), atrial fibrillation (A-Fib), prior myocardial infarction (MI), hypertension (HTN), hyperlipidemia (HLD), and osteoarthritis. Other preoperative variables collected included alcohol use, smoking status, and home narcotic use.

Intraoperative variables included number of fusion levels, operative time, estimated blood loss (EBL), administration of packed red blood cell (PRBC) or cell-saver transfusions, and whether an osteotomy was performed. Other operative variables assessed included use of somatosensory stimulus evoked potentials (SSEP), transcranial motor evoked potentials (TcMEP), electromyography (EMG), and fluoroscopy. Additionally, whether patients received bone graft and intra-operative drain placement were also collected. Intraoperative complications collected included spinal cord injury, nerve root injury, and incidental durotomy.

Postoperative complications included length of stay in hospital (LOS), ICU transfer rate, delirium, urinary tract infection (UTI), fever, ileus, deep and superficial surgical site infection (SSI), wound dehiscence, draining wounds, pneumonia, hypertension (HTN), hypotension, hematoma, anemia, MI, weakness, sensory deficit, and urinary retention.
Baseline and post-operative inpatient patient-reported pain scores and ambulatory status were also collected. Pain scores were recorded on a scale from 0 to 10 first post-operative day and prior to discharge. Ambulatory status included the number of days from the operating room to ambulation, the number of steps of first ambulatory steps, and the number of steps of last ambulatory steps.

Parametric data were expressed as means \pm standard deviation (SD) and compared using the Student's t-test. Nonparametric data were expressed as median [interquartile range] and compared via the Mann-Whitney $U$ test. Nominal data were compared with the $\chi^{2}$ test. A multivariate nominal logistic regression was used to assess the association between age and patient-reported pain scores. All tests were two-sided and were statistically significant if the $\mathrm{p}$-value was less than 0.05. Statistical analysis was performed using JMP ${ }^{\oplus}$, Version 13. SAS Institute Inc., Cary, NC, 1989-2007.

\section{RESULTS}

\section{Patient Demographics and Preoperative Variables}

There were 92 adults ( $\geq 18$ years old) who met the inclusion criteria of this study (Elderly: $n=43$; Young: $n=49$ ), Table 1. Overall, the average age for the Elderly cohort was $71.6 \pm 4.7$ years and for the Young cohort was $41.4 \pm 16.8$ years. There were no significant differences in gender or BMI between the cohorts (Elderly: 26.8 $\pm 4.3 \mathrm{~kg} / \mathrm{m}^{2}$ vs. Young: $41.4 \pm 16.8 \mathrm{~kg} / \mathrm{m}^{2}, p=0.7759$ ), Table 1 . The prevalence of some comorbidities between the cohorts were similar, including depression $(p=0.5103)$, anxiety $(0.7253), \operatorname{COPD}(p=0$. 3116), diabetes $(p=0.3733), \mathrm{CHF}(p=0.3729), \mathrm{CAD}(p=0.0656)$, A-Fib $(p=0.1253)$, and prior MI $(p=0.3116)$. The Elderly cohort had a higher rate of HTN (Elderly: $72.1 \%$ vs. Young: $34.7 \%, p=0.0003$ ), HLD (Elderly: $55.8 \%$ vs. Young: $24.5 \%, p=0.0021$ ), and osteoarthritis (Elderly: $53.5 \%$ vs. Young: $16.3 \%, p=0.0002$ ) but a lower percentage of alcohol use (Elderly: $41.9 \%$ vs. Young: $20.4 \%, p=0.0257)$. Current smoking $(p=0.4929)$ and pre-operative narcotic use $(p=0.2971) \mathrm{did}$ not differ between the cohorts, Table 1 .

\section{Intraoperative Variable and Complications}

The median number of fusion levels (Elderly: 9 [7-10] vs. Young: 9 [7-13], $p=0.2844$ ) and operative time (Elderly: $339.3 \pm 147.0$ mins $v$ s. Young: $312.7 \pm 103.1, p=0.3232)$ were similar between cohorts, Table 2. The Young cohort had a higher rate of SSEP (Elderly: $25.0 \% v s$. Young: $46.5 \%, p=0.0415$ ) and TcMEP (Elderly: $7.5 \%$ vs. Young: $34.9 \%$, $p=0.0025)$, but the utilization of EMG $(p=0.8070)$ and fluoroscopy $(p=0.1064)$ were similar between cohorts, Table 2 . The Elderly cohort also had a higher rate of PRBC Transfusions (Elderly: 70.0\% vs. Young: $38.8 \%, p=0.0030$ ), Table 2 . There were no significant differences in the other surgical variables, including intra-operative EBL (Elderly: $1544.8 \pm 1265.0 \mathrm{~mL}$ vs. Young: $1384.2 \pm 1521.1 \mathrm{~mL}, p=0.5819$ ), cellsaver transfusions (Elderly: $76.7 \%$ vs. Young: $67.4 \%, p=0.3179$ ), and the performance of osteotomy (Elderly: $18.6 \%$ vs. Young: $14.3 \%, p=$ 0.5758 ) between the cohorts, Table 2 . There were also no significant differences in nerve root/spinal cord injuries $(p=0.000)$ or incidental durotomy $(p=0.5854)$, Table 2 . The proportion of patients receiving bone graft $(p=0.1732)$ and having a drain placement $(p=0.8986)$ were also similar between the cohorts, Table 2 . 
Aladine A. Elsamadicy (2019) Association Between Age and Short-Term Patient Reported Pain Scores After Complex Spinal Fusion for Adult Deformity Correction: Is Perception of Pain Different?

Table 1. Demographic and Comorbidities

\begin{tabular}{|c|c|c|c|}
\hline Variables & $\begin{array}{l}\text { Elderly } \\
(\mathrm{n}=43)\end{array}$ & $\begin{array}{l}\text { Young } \\
(n=49)\end{array}$ & P-Value \\
\hline Female $(\%)$ & 72.1 & 65.3 & 0.4845 \\
\hline Age (Years) & $71.6 \pm 4.7$ & $41.4 \pm 16.8$ & $<0.0001^{*}$ \\
\hline BMI $\left(\mathrm{kg} / \mathrm{m}^{2}\right)$ & $26.8 \pm 4.3$ & $27.2 \pm 7.2$ & 0.7759 \\
\hline Depression (\%) & 30.2 & 36.7 & 0.5103 \\
\hline Anxiety (\%) & 25.6 & 22.5 & 0.7253 \\
\hline COPD $(\%)$ & 9.3 & 4.1 & 0.3116 \\
\hline Diabetes (\%) & 14.0 & 8.2 & 0.3733 \\
\hline CHF (\%) & 2.3 & 6.1 & 0.3729 \\
\hline CAD (\%) & 18.6 & 6.1 & 0.0656 \\
\hline A-Fib (\%) & 9.3 & 2.0 & 0.1253 \\
\hline Prior MI (\%) & 9.3 & 4.1 & 0.3116 \\
\hline HTN (\%) & 72.1 & 34.7 & $0.0003^{*}$ \\
\hline HLD (\%) & 55.8 & 24.5 & $0.0021^{*}$ \\
\hline Osteoarthritis (\%) & 53.5 & 16.3 & $0.0002 *$ \\
\hline Alcohol Use (\%) & 41.9 & 20.4 & $0.0257^{*}$ \\
\hline Current Smoker (\%) & 11.6 & 16.7 & 0.4929 \\
\hline Pre-Op Narcotic Use (\%) & 54.8 & 43.8 & 0.2971 \\
\hline
\end{tabular}

Table 2. Intraoperative Variables and Complications

\begin{tabular}{|l|c|c|c|}
\hline \multicolumn{1}{|c|}{ Variables } & $\begin{array}{c}\text { Elderly } \\
(\mathbf{n}=\mathbf{4 3})\end{array}$ & $\begin{array}{c}\text { Young } \\
(\mathbf{n = 4 9 )}\end{array}$ & P-Value \\
\hline Median \# of Levels [IQR] & $9[7-10]$ & $9[7-13]$ & 0.2844 \\
\hline Osteotomy (\%) & 18.6 & 14.3 & 0.5758 \\
\hline SSEP (\%) & 25.0 & 46.5 & $0.0415^{*}$ \\
\hline TcMEP (\%) & 7.5 & 34.9 & $0.0025^{*}$ \\
\hline EMG (\%) & 25.0 & 22.7 & 0.8070 \\
\hline Fluoroscopy (\%) & 50.0 & 67.4 & 0.1064 \\
\hline Bone Graft (\%) & 88.4 & 95.9 & 0.1732 \\
\hline Operative Time (mins) & $339.3 \pm 147.0$ & $312.7 \pm 103.1$ & 0.3232 \\
\hline EBL (mL) & $1544.8 \pm 1265.0$ & $1384.2 \pm 1521.1$ & 0.5819 \\
\hline PRBC Transfusions (\%) & 70.0 & 38.8 & $0.0030 *$ \\
\hline $\begin{array}{l}\text { Cell Saver Transfusions } \\
\text { (\%) }\end{array}$ & 76.7 & 67.4 & 0.3179 \\
\hline Drain Placement (\%) & 8.3 .4 & 8.3 & 0.8 \\
\hline $\begin{array}{l}\text { Nerve/Spinal Cord } \\
\text { Damage (\%) }\end{array}$ & 0.0 & 0.0 & 0.000 \\
\hline Durotomy (\%) & & & \\
\hline
\end{tabular}

SSEP $=$ Sensory Stimulus Evoked Potentials; TcMEP = Transcranial Motor Evoked Potentials;

\section{Postoperative Complications}

There were no significant differences in overall LOS between the cohorts (Elderly: $8.3 \pm 5.4$ days vs. Young: $6.4 \pm 3.1$ days, $p=0.0525$ ) or ICU transfer (Elderly: $56.1 \%$ vs. Young: $50.0 \%, p=0.5657$ ), Table 3. Compared to the Elderly group, the Elderly cohort experienced a significantly higher incidence of post-operative delirium (Elderly: 16.3\% vs. Young: $0.0 \%, p=0.0033$ ) and anemia (Elderly: $58.1 \%$ vs. Young: $29.2 \%, p=0.0053$ ), Table 3. There were no significant differences in the incidence of other post-operative complications, including UTI ( $p=0.1314)$, fever $(p=0.2102)$, ileus $(p=0.4929)$, Deep SSI (0.2437), wound dehiscence $(p=0.5053)$, draining wounds $(p=$ $0.1349)$, superficial SSI $(p=0.3476)$, pneumonia $(p=0.3412)$, HTN ( $p=0.0670)$, hypotension $(p=0.8379)$, hematoma $(p=0.9373)$, MI ( $p=0.1308)$, weakness $(p=0.8084)$, sensory deficits $(p=0.000)$, and urinary retention $(p=0.2093)$, Table 3 .

Table 3. Postoperative Complications

\begin{tabular}{|c|c|c|c|}
\hline Variables & $\begin{array}{l}\text { Elderly } \\
(\mathrm{n}=\mathbf{4 3})\end{array}$ & $\begin{array}{l}\text { Young } \\
(\mathrm{n}=49)\end{array}$ & P-Value \\
\hline LOS (Days) & $8.3 \pm 5.4$ & $6.4 \pm 3.1$ & 0.0525 \\
\hline ICU Transfer (\%) & 56.1 & 50.0 & 0.5657 \\
\hline Delirium (\%) & 16.3 & 0.0 & $0.0033^{*}$ \\
\hline UTI (\%) & 9.3 & 2.1 & 0.1314 \\
\hline Fever (\%) & 4.9 & 12.5 & 0.2102 \\
\hline Ileus (\%) & 11.6 & 16.7 & 0.4929 \\
\hline Deep SSI (\%) & 7.3 & 2.1 & 0.2437 \\
\hline Wound Dehiscence (\%) & 4.7 & 2.1 & 0.5053 \\
\hline Draining Wound (\%) & 4.7 & 0.0 & 0.1349 \\
\hline Superficial SSI (\%) & 0.0 & 2.1 & 0.3476 \\
\hline Pneumonia (\%) & 0.0 & 2.1 & 0.3412 \\
\hline Hypertension (\%) & 11.6 & 2.1 & 0.0670 \\
\hline Hypotension (\%) & 14.0 & 12.5 & 0.8379 \\
\hline Hematoma (\%) & 2.3 & 2.1 & 0.9373 \\
\hline Anemia (\%) & 58.1 & 29.2 & $0.0053^{*}$ \\
\hline MI (\%) & 4.7 & 0.0 & 0.1308 \\
\hline Weakness (\%) & 7.0 & 8.3 & 0.8084 \\
\hline Sensory Deficit (\%) & 0.0 & 0.0 & 0.0000 \\
\hline Urinary Retention (\%) & 2.3 & 8.3 & 0.2093 \\
\hline
\end{tabular}

Pre- and Post-Operative Patient Reported Pain Scores and Ambulatory Status

Baseline pain scores (Elderly: $6.0 \pm 2.7$ vs. Young: $5.1 \pm 3.5, p=$ 0.1885 ) as well as the first pain score (Elderly: $6.4 \pm 3.0$ vs. Young: $5.8 \pm$ 2.7, $p=0.3331$ ) and the last pain score (Elderly: $3.5 \pm 3.5$ vs. Young: 4.4 $\pm 3.1, p=0.1990$ ) were similar between both cohorts, Table 4 . There were no significant differences between the change from baseline to 
first pain score (Elderly: $+0.44 \pm 3.4$ vs. Young: $+0.71 \pm 3.8, p=0.7180$ ), but the elderly trended to have a greater reduction of pain (Elderly: $-2.4 \pm 4.1$ vs. Young: $-0.69 \pm 4.5, p=0.0556$ ), Table 4. Additionally, there were no significant differences in the number of days from the operating room to ambulation $(p=0.9904)$ or the number of steps of last ambulatory steps $(p=0.0789)$, Table 4 . However, the Elderly cohort had significantly fewer ambulatory steps on the first postoperative ambulatory day compared to Young cohort (Elderly: $40.2 \pm$ 63.4 vs. Young: $106.0 \pm 134.6, p=0.0042)$, Table 4.

Table 4. Pre- and Post-Operative Patient Reported Pain Scores and Ambulatory Status

\begin{tabular}{|c|c|c|c|}
\hline Variables & $\begin{array}{l}\text { Elderly } \\
(\mathrm{n}=43)\end{array}$ & $\begin{array}{l}\text { Young } \\
(\mathrm{n}=49)\end{array}$ & P-Value \\
\hline \multicolumn{4}{|c|}{ Pain Scores } \\
\hline Baseline Pain Score & $6.0 \pm 2.7$ & $5.1 \pm 3.5$ & 0.1885 \\
\hline First Pain Score & $6.4 \pm 3.0$ & $5.8 \pm 2.7$ & 0.3331 \\
\hline Last Pain Score & $3.5 \pm 3.5$ & $4.4 \pm 3.1$ & 0.1990 \\
\hline$\Delta$ Baseline-First Pain Score & $+0.44 \pm 3.4$ & $+0.71 \pm 3.8$ & 0.7180 \\
\hline$\Delta$ Baseline-Last Pain Score & $-2.4 \pm 4.1$ & $-0.69 \pm 4.5$ & 0.0556 \\
\hline \multicolumn{4}{|c|}{ Ambulatory Status } \\
\hline $\begin{array}{l}\text { Days from OR to Ambulation } \\
\text { (Days) }\end{array}$ & $2.0 \pm 1.4$ & $2.0 \pm 1.3$ & 0.9904 \\
\hline $\begin{array}{l}\text { \# of Steps of First Ambulatory } \\
\text { Steps (ft) }\end{array}$ & $40.2 \pm 63.4$ & $\begin{array}{c}106.0 \pm \\
134.6\end{array}$ & $0.0042 *$ \\
\hline $\begin{array}{l}\text { \# of Steps of Last Ambulatory } \\
\text { Steps (ft) }\end{array}$ & $189.1 \pm 153.3$ & $\begin{array}{c}271.7 \pm \\
278.1\end{array}$ & 0.0789 \\
\hline
\end{tabular}

\section{DISCUSSION}

In this retrospective study, we show that elderly patients $(\geq 65$ years old) had a greater pain reduction compared to younger patients after complex spinal fusions ( $\geq 5$ levels) for adult deformity correction.

Previous studies have demonstrated associations between age and complications after adult deformity correction surgery. In a retrospective review of 206 patients undergoing spinal fusion for scoliosis correction, Smith et al. found that total perioperative complications were significantly higher in the 65-85 year age range than in 25-44 and 44-85 year age ranges [10]. Similarly, in another retrospective analysis of 46 patients who underwent a thoracic or lumbar arthrodesis ( $\geq 5$ spinal levels), Daubs et al. demonstrated that patients older than age 69 years had a 9-fold greater likelihood to have a major complication [12]. In another retrospective review of a prospective multicenter study of 480 patients who underwent spinal surgery for deformity correction, Soroceanu et al. demonstrated that older age trended to be a predictor for a higher medical complication rate [14]. Analogous to the aforementioned studies, our study showed that elderly patients had increasing rates of post-operative anemia and incidences of delirium compared to the younger cohort.

Along with complication rates, previous studies have explored the impact age has on PROs after deformity correction surgery. In the Smith et al. study of 206 patients, the authors showed that elderly patients had initial greater disability and greater neck and back pain [10]. In a retrospective study of 55 patients who underwent $\geq 5$ levels of spinal fusion to the sacrum with iliac fixation, Elsamadicy et al. found no significant difference in pain scores between young and elderly cohorts after surgery[13]. Similarly, in the retrospective analysis of 46 patients who underwent a thoracic or lumbar arthrodesis ( $\geq 5$ spinal levels), Daubs et al. reported that age had no impact on disability (ODI) scores [12]. Our study showed that elderly patients trended to have worse pain scores before spinal fusion, but the surgery brought a greater improvement of pain than the younger cohort by their last day in the hospital. This suggests that elderly people have better patientreported outcomes and that spinal surgery may be more beneficial for them than the younger cohort.

Previous studies have also looked at the impact of age on long-term patient-reported outcomes collected during followup. In a retrospective review of 374 patients who had undergone a 3-column pedicle subtraction osteotomy, Scheer et al. showed that the older patients had greater improvement in 2-year disability and Scoliosis Research Society-22 questionnaire (SRS) total scores [9]. Furthermore, disability scores and leg pain at 2-year follow up were significantly more improved among elderly patients than younger ones [9]. In contrast, in a multicenter prospective study of 56 patients who underwent primary spinal deformity surgery for scoliosis, Birdwell et al. found that age had no effect on rates of improvement in pain in a 2-year follow-up [11]. In a prospective study of 40 patients with posterior reconstruction with an instrumented fusion from the thoracic spine to the sacrum, Crawford et al. demonstrated that the elderly cohort had worse Physical Component Score (PCS) but higher MCS scores at baseline [17]. However, at two-year follow-up, there were no significant differences in any of the scores [17].

In an era of trying to reduce medical costs, a few studies have looked at the impact of age and pain on cost following surgery. In a retrospective analysis of 76 US patients undergoing spinal fusion ( $\geq 5$ levels) for deformity correction, Yagi et al. reported that direct hospital costs for the initial surgery averaged to $\$ 71,638 \pm 23,246$ and 2-year follow up costs came out to be $\$ 44,479 \pm 10,943$ [18]. In a retrospective study of a prospective, consecutive, multicenter database of 514 patients who underwent surgery for adult spinal deformity, Fischer et al. demonstrated that age greater than 55 years was associated with cost-effectiveness, as measured by cost/QALY [19]. Two important factors that affect cost are pain medication use and hospital length of stay. In a retrospective study of 78 postoperative patients requiring morphine, Macintyre et al. found that the strongest correlator with increased morphine requirement was increasing age [8]. In a prospective longitudinal study of 752 patients who underwent an elective laminectomy and fusion for degenerative lumbar conditions, Sivaganesan et al. found that the average 90-day cost of surgery was $\$ 29,295$, and the amount of preop and postop opioid use was a significant driver of that cost [20]. Concerning length of stay, in a retrospective study of 55 patients who underwent $\geq 5$ levels of spinal fusion to the sacrum with iliac fixation, Elsamadicy et al. found no difference length of stay between young and elderly cohorts [13]. In contrast, in a prospective cohort study of 411 patients admitted to a New York hospital with a hip fracture, Morrison et al. 
found that greater pain is associated with longer length of stay and long-term functional impairment, both of which increases costs [21]. Analogously, in the Romano et al. study of 10,416 patients, the authors reported that patients older than 70 years of age were had a 1.8 times longer length of stay those 31-40 years of age [16]. In a retrospective study of 480 patients undergoing surgery for adult spinal deformity, McCarthy et al. found that a 1-year increase in patient age increased index costs by $\$ 2,600$, and an extra day in the hospital increased index costs by $\$ 4,600$ [22]. Thus, reducing pain, and therefore pain medication use, and hospital length of stay would help drive down the costs of complex spinal surgery.

This study has limitations with potential implications for study interpretation. Although all variables were recorded pre-, peri-, and postoperatively, they were reviewed retrospectively and, as such, are limited by the weaknesses inherent to retrospective analyses. Furthermore, a relatively small patient sample size from only one academic center was used, making broad conclusions difficult and potentially biasing our results for particular patient population or treatment paradigms. Despite these limitations, this study has demonstrated that an elderly age is associated with a greater reduction in pain after complex spinal fusion ( $\geq 5$ levels) for deformity correction.

\section{CONCLUSIONS}

Our study suggests that age may have an impact in patient perception of pain and improvement after complex spinal fusions ( $\geq 5$ levels). Consideration of patients' age may facilitate tailored pain management and physical therapy regimens in deformity patients undergoing correction surgery.

\section{References}

1. McGirt MJ, Parker SL, Asher AL, Norvell D, Sherry N, Devin CJ. (2014) Role of prospective registries in defining the value and effectiveness of spine care. Spine (Phila Pa 1976). 39(22 Suppl 1): S117-128. [Crossref]

2. Black N. (2013) Patient reported outcome measures could help transform healthcare. $B M J$ (Clinical research ed). 346: f167. [Crossref]

3. Yamashita K, Ohzono K, Hiroshima K. (2006) Patient satisfaction as an outcome measure after surgical treatment for lumbar spinal stenosis: testing the validity and discriminative ability in terms of symptoms and functional status. Spine (Phila Pa 1976). 31(22): 2602-2608. [Crossref]

4. Di Capua J, Lugo-Fagundo N, Somani S, et al. (2018) Diabetes Mellitus as a Risk Factor for Acute Postoperative Complications Following Elective Adult Spinal Deformity Surgery. Global Spine J. 8(6): 615-621. [Crossref]

5. Cowan JA, Jr., Dimick JB, Wainess R, Upchurch GR, Jr., Chandler WF, La Marca F. (2006) Changes in the utilization of spinal fusion in the United States. Neurosurgery. 59(1): 15-20; discussion 15-20. [Crossref]

6. De Benedittis S, Lorenzetti A, Migliore M, Spagnoli D, Tiberio F, Villani RM. (1996) Postoperative Pain in Neurosurgery: A Pilot Study in Brain Surgery. Neurosurgery. 38(3): 466-470. [Crossref]

7. Wallace MS, Wallace AM, Lee J, Dobke MK. (1996) Pain after breast surgery: a survey of 282 women. PAIN®. 66(2): 195-205. [Crossref]

8. Macintyre PE, Jarvis DA. (1996) Age is the best predictor of postoperative morphine requirements. Pain. 64(2): 357-364. [Crossref]

9. Scheer J, Lafage V, Smith J, et al. Impact of age on the likelihood of reaching a minimum clinically important difference in 374 three-column spinal osteotomies: Clinical article. Vol 202014. [Crossref]

10. Smith JS, Shaffrey CI, Glassman SD, et al. (2011) Risk-Benefit Assessment of Surgery for Adult Scoliosis: An Analysis Based on Patient Age. Spine. 36(10): 817-824. [Crossref]

11. Bridwell KH, Berven S, Glassman S, et al. (2007) Is the SRS-22 Instrument Responsive to Change in Adult Scoliosis Patients Having Primary Spinal Deformity Surgery? Spine. 32(20): 2220-2225. [Crossref]
12. Daubs MD, Lenke LG, Cheh G, Stobbs G, Bridwell KH. (2007) Adult Spinal Deformity Surgery: Complications and Outcomes in Patients Over Age 60. Spine. 32(20): 2238-2244. [Crossref]

13. Elsamadicy AA, Adogwa O, Sergesketter A, et al. (2017) Impact of Age on Change in Self-Image 5 Years After Complex Spinal Fusion ( $\geq 5$ Levels). World neurosurgery. 97: 112-116. [Crossref]

14. Soroceanu A, Burton DC, Oren JH, et al. (2016) Medical Complications After Adult Spinal Deformity Surgery: Incidence, Risk Factors, and Clinical Impact. Spine (Phila Pa 1976). 41(22): 1718-1723. [Crossref]

15. Wang MC, Chan L, Maiman DJ, Kreuter W, Deyo RA. (2007) Complications and mortality associated with cervical spine surgery for degenerative disease in the United States. Spine (Phila Pa 1976). 32(3): 342-347. [Crossref]

16. Romano PS, Campa DR, Rainwater JA. (1997) Elective Cervical Discectomy in California: Postoperative In-Hospital Complications and Their Risk Factors. Spine. 22(22): 2677-2692. [Crossref]

17. Crawford CH, Carreon LY, Bridwell KH, Glassman SD. (2012) Long fusions to the sacrum in elderly patients with spinal deformity. European Spine Journal. 21(11): 2165-2169. [Crossref]

18. Yagi M, Ames CP, Keefe M, et al. (2018) A cost-effectiveness comparisons of adult spinal deformity surgery in the United States and Japan. European Spine Journal. 27(3): 678-684. [Crossref]

19. Fischer CR, Terran J, Lonner B, et al. (2014) Factors Predicting Cost-effectiveness of Adult Spinal Deformity Surgery at 2 Years. Spine Deformity. 2(5): 415-422. [Crossref]

20. Sivaganesan A, Chotai S, Parker SL, McGirt MJ, Devin CJ. (2018) Drivers of Variability in 90-Day Cost for Elective Laminectomy and Fusion for Lumbar Degenerative Disease. Neurosurgery. [Crossref]

21. Morrison RS, Magaziner J, McLaughlin MA, et al. (2003) The impact of postoperative pain on outcomes following hip fracture. Pain. 103(3): 303-311. [Crossref]

22. McCarthy IM, Hostin RA, Ames CP, et al. (2014) Total hospital costs of surgical treatment for adult spinal deformity: an extended follow-up study. The Spine Journal. 14(10): 2326-2333. [Crossref]

\section{Citation:}

Aladine A. Elsamadicy, Megan Lee, Andrew Koo, Adam J. Kundishora, Joaquin Camara-Quintana, Tariq Qureshi, Luis Kolb, Maxwell Laurans, Khalid Abbed, Isaac O. Karikari (2019) Association Between Age and Short-Term Patient Reported Pain Scores After Complex Spinal Fusion for Adult Deformity Correction: Is Perception of Pain Different?. J Clin Res Med Volume 2(2): 1-5. 